\title{
Aortic valve endocarditis complicated by proximal false aneurysm
}

\author{
Pietro Giorgio Malvindi ${ }^{1}$, Elisa Mikus ${ }^{2}$, Luca Caprili ${ }^{3}$, Giuseppe Santarpino ${ }^{4}$, Vito Margari ${ }^{1}$, \\ Simone Calvi ${ }^{2}$, Giuseppe Nasso ${ }^{5}$, Renato Gregorini ${ }^{4}$, Carmine Carbone ${ }^{1}$, Alberto Albertini ${ }^{2}$, \\ Giuseppe Speziale ${ }^{5}$, Domenico Paparella ${ }^{1,6}$
}

${ }^{1}$ Cardiac Surgery, Santa Maria Hospital, Bari, Italy; ${ }^{2}$ Cardiac Surgery, Maria Cecilia Hospital, Cotignola, Italy; ${ }^{3}$ Cardiac Surgery, Salus Hospital, Reggio Emilia, Italy; ${ }^{4}$ Cardiac Surgery, Città di Lecce Hospital, Lecce, Italy; ${ }^{5}$ Cardiac Surgery, Anthea Hospital, Bari, Italy; ${ }^{6}$ Department of Emergency and Organ Transplant, University of Bari Aldo Moro, Bari, Italy

Correspondence to: Pietro Giorgio Malvindi. Department of Cardiac Surgery, Santa Maria Hospital, Via de Ferrariis 22, Bari 70100, Italy.

Email: pg.malvindi@hotmail.com.

\begin{abstract}
Background: Aortic valve endocarditis remains a life-threatening condition, especially in cases of periannular complications. Aorto-ventricular discontinuity associated with proximal false aneurysm represents a severe picture caused by extensive tissue disruption and is usually associated with prosthetic valve infection. Complex surgical repair is required in these cases and continues to be associated with high mortality and morbidity rates.
\end{abstract}

Methods: We retrieved information for 32 patients undergoing operation for infective aortic valve/ prosthetic valve endocarditis complicated by pseudoaneurysm arising from aorto-ventricular discontinuity. Patients were relatively young, mostly male and most of them had a prior cardiac operation. Aortic root replacement with valve graft conduit was performed in all cases; it was associated with other procedures in seven patients: CABG $(n=2), M V$ surgery $(n=3), M V$ surgery + CABG $(n=1)$ and pulmonary valve replacement $(\mathrm{n}=1)$. We reported and analysed patient outcomes at early and mid-term follow-up.

Results: Pre-discharge mortality was $22 \%(n=7)$. The postoperative course was complicated in $24(75 \%)$ cases. Eighteen patients (56\%) sustained low cardiac output resulting in multiple organ failure syndrome and death in five cases. One patient (3\%) experienced a major neurologic deficit with a permanent cerebral stroke. Acute kidney injury complicated the course in 12 cases (37\%), continuous renal replacement therapy was necessary in four patients (12\%). Overall survival and freedom from endocarditis and reoperation at 5-year was $59 \%$ and $89 \%$, respectively.

Conclusions: Patients with complicated aortic valve endocarditis presented generally in a poor preoperative state. Surgical treatment poses a non-negligible risk of postoperative mortality and morbidity but provides an acceptable survival rate and a satisfactory recovery at mid-term.

Keywords: Endocarditis; aorta; aortic valve; aortic root; reoperation

Submitted Nov 10, 2018. Accepted for publication May 24, 2019.

doi: $10.21037 /$ acs.2019.05.17

View this article at: http://dx.doi.org/10.21037/acs.2019.05.17

\section{Introduction}

Aortic valve endocarditis remains a life-threatening condition that is still burdened by significant mortality and morbidity rates. Involvement of the surrounding tissue has been described in about $20 \%$ of cases (1) and is more frequent in cases of Staphylococcus aureus infection and in prosthetic valve endocarditis (2). The local extension of the infective process causes tissue destruction and periannular abscess formation. The progression of the pathology may further complicate the scenario, with fistulisation in other cardiac structures or the development of a pseudoaneurysm (3). Surgical therapy is invariably required in these cases and represents the only therapeutic option, but it is associated with high postoperative mortality and morbidity $(2,4,5)$. In this paper, we describe our experience 
in the treatment of aortic valve/aortic root endocarditis complicated by extensive annular and periannular tissue disruption.

\section{Methods}

\section{Patient population}

Clinical and administrative databases of four Italian cardiac centres were searched for the period January 2011December 2017. Search results returned 218 patients operated on for aortic valve endocarditis. Surgical notes and preoperative imaging data were reviewed. Thirty-two patients underwent surgery for endocarditis complicated by proximal false aneurysm and were included in this analysis. Because of the retrospective nature of this study, the local Ethics Committee waived the need for patient consent.

The mean age of the patients at the time of operation was $60 \pm 15$ years (range, $27-82$ years) and 30 (94\%) were male. In 28 out of 32 cases, there was evidence of active infective endocarditis defined as positive blood or tissue cultures, signs of ongoing sepsis or progression of the lesion. Eighteen patients required an urgent operation, in four cases the surgical procedure was in an emergency setting. Table 1 reports patients' characteristics.

Twenty-eight patients had undergone prior cardiac surgery, three of them had already received two operations; the median interval between the previous procedure and the actual operation was 22 months (range, 1-166 months). All of these patients had an aortic valve prosthesis, fifteen of them as a composite valve graft after aortic root replacement. Table 2 details prior operation data.

\section{Indication for reoperation and surgical techniques}

Echocardiography and computed tomography (CT) scan were performed in all patients. Aorto-ventricular discontinuity was defined intraoperatively as a separation between the aorta and left ventricle of more than one-third of its circumference (3). The disruption of aorto-ventricular tissue led to the formation of aortic pseudoaneurysm, defined as a cavity of variable extension and position contained by the mediastinal surrounding structures (6) and in communication with the cardiovascular lumen. Typical imaging characteristics are ultrasound signs of flow in its interior and pulsatile pouch, and CT evidence of contrast medium filling $(7,8)$.

Aortic root replacement was performed in all patients.

\begin{tabular}{|c|c|}
\hline Variables & $\begin{array}{l}\text { N. of patients } \\
\text { or mean } \pm \text { SD (\%) }\end{array}$ \\
\hline N. patients & 32 \\
\hline Male/female & $30 / 2(94 \% / 6 \%)$ \\
\hline Age (years) & $60 \pm 15$ \\
\hline \multicolumn{2}{|l|}{ Presentation } \\
\hline NYHA III & $13(41 \%)$ \\
\hline NYHA IV & $5(16 \%)$ \\
\hline Chest pain & $6(19 \%)$ \\
\hline Syncope & $3(9 \%)$ \\
\hline Ongoing cardiogenic shock & $2(6 \%)$ \\
\hline New coronary artery disease & $2(6 \%)$ \\
\hline Active endocarditis & 27 (84\%) \\
\hline LVEF $>50$ & $27(84 \%)$ \\
\hline LVEF [30-50] & $5(16 \%)$ \\
\hline Preoperative inotropes & $4(13 \%)$ \\
\hline Preoperative mechanical ventilation & $3(9 \%)$ \\
\hline \multicolumn{2}{|l|}{ Cardiovascular risk factors } \\
\hline Known coronary artery disease & $2(6 \%)$ \\
\hline Peripheral vascular disease & $2(6 \%)$ \\
\hline Diabetes mellitus & $5(16 \%)$ \\
\hline Hypertension & $18(56 \%)$ \\
\hline Hypercholesterolemia & $14(44 \%)$ \\
\hline Smoking history & $16(50 \%)$ \\
\hline Atrial fibrillation & $3(9 \%)$ \\
\hline Permanent pace-maker & $2(6 \%)$ \\
\hline Haemo-dialysis & $1(3 \%)$ \\
\hline COPD & $1(3 \%)$ \\
\hline Previous cerebral stroke & $5(16 \%)$ \\
\hline Urgent/emergency setting & $22(69 \%)$ \\
\hline Interval admission to operation (days) & $4 \pm 3.6$ \\
\hline
\end{tabular}

Concomitant coronary artery bypass graft (CABG) was planned based on preoperative angiography in two patients. Table 3 describes the actual operative procedures performed.

A median sternotomy was performed in all cases. The 


\begin{tabular}{ll}
\hline Table 2 Prior operation performed & \\
\hline Variables & $\begin{array}{l}\text { N. of patients or } \\
\text { median [range] (\%) }\end{array}$ \\
\hline Previous cardiac operation & $28(88 \%)$ \\
\hline Interval prior-actual operation (months) & $22[1-166]$ \\
\hline Patients with one previous operation & $25(78 \%)$ \\
ARR & $14(44 \%)$ \\
AVR & $8(25 \%)$ \\
AVR + MVr + atrial fibrillation ablation & $1(3 \%)$ \\
AVR + MVR + ascending aorta & $1(3 \%)$ \\
replacement & \\
AVR + ascending aorta replacement & $1(3 \%)$ \\
\hline Patients with two previous operations & $3(9 \%)$ \\
\hline AVR + CABG \& AVR & $1(3 \%)$ \\
AVR \& AVR & $1(3 \%)$ \\
ARR \& MVR + CABG & $1(3 \%)$ \\
\hline ARR, aortic root replacement (all patients had Bentall procedures); \\
AVR, aortic valve replacement; MVr, mitral valve repair; MVR, \\
mitral valve replacement.
\end{tabular}

common femoral artery $(52 \%)$ or ascending aorta/aortic arch $(44 \%)$ were used as arterial inflow and the right atrium (directly or via the femoral vein using a double staged cannula) as venous site of drainage. In 4 patients cardiopulmonary bypass (CPB) was started before chest reentry and in 2 of them, deep cooling and a short period of deep hypothermic circulatory arrest was necessary before opening a pseudoaneurysm involving all of the ascending aorta. Reasons for starting cardiopulmonary bypass before re-sternotomy were: presence of a giant pseudoaneurysm adherent to the posterior aspect of the sternum $(n=3)$ (Figure 1) and in one patient, compression and obliteration of the valve graft conduit caused by a pseudoaneurysm arising from the non-coronary/right coronary aortoventricular discontinuity. In this last case, a left minithoracotomy allowed vent insertion through the left ventricular apex before chest re-entry. We did not experience false aneurysm rupture during chest re-entry.

Cold blood cardioplegia was administered in most of the cases $(85 \%)$, usually into the ascending aorta/vascular prosthesis and selectively into the coronary ostia.

Coronary reimplantation using the coronary button technique was planned in all patients. Unplanned CABG

\begin{tabular}{|ll}
\hline Table 3 Operative data & $\begin{array}{l}\text { N. of patients or } \\
\text { mean } \pm \text { SD }(\%)\end{array}$ \\
\hline Variables & $25(78 \%)$ \\
\hline ARR & $1(3 \%)$ \\
\hline ARR + CABG & $1(3 \%)$ \\
\hline ARR + CABG + VSD closure & $2(6 \%)$ \\
\hline ARR + MVr & $1(3 \%)$ \\
\hline ARR + MVr + CABG & $1(3 \%)$ \\
\hline ARR + MVR + CABG & $1(3 \%)$ \\
\hline ARR + PVR & $2(6 \%)$ \\
\hline Unplanned CABG & $226 \pm 100$ \\
\hline CPB time & $170 \pm 48$ \\
\hline Xclamp time & \\
\hline ARR, aortic root replacement (all patients had Bentall procedures); \\
AVR, aortic valve replacement; MVr, mitral valve repair; MVR, \\
mitral valve replacement; PVR, pulmonary valve replacement; \\
VDS, ventricular septal defect.
\end{tabular}

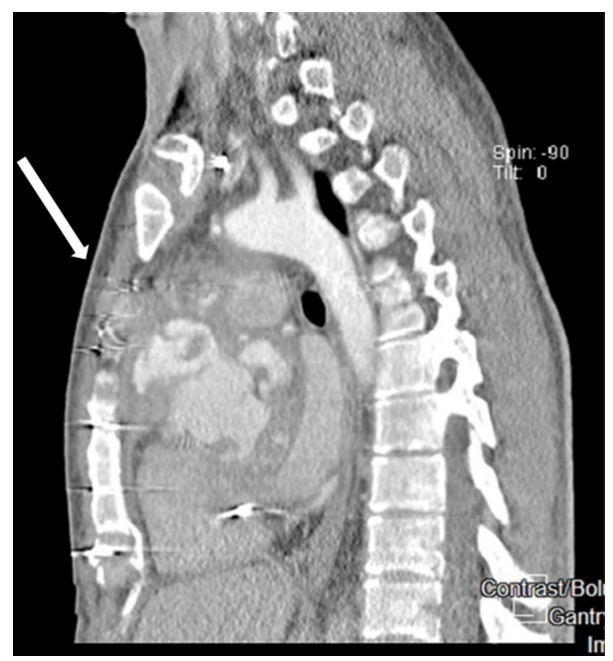

Figure 1 Multiplanar CT image of a giant false aneurysm arising from proximal anastomosis of valve graft conduit and extending to the posterior aspect of the sternum (white arrow).

was necessary in 2 patients: right internal mammary artery (RIMA) on right coronary artery (RCA) for right ventricular (RV) failure during $\mathrm{CPB}$ weaning attempt, saphenous vein graft (SVG) on left anterior descending (LAD) and RCA for failure in coronary ostia reimplantation. 
As for all infected endocarditis cases, the surgical strategy provided radical debridement of all infective tissue with removal of necrotic tissue and prosthetic material. In thirteen patients, an extensive annular reconstruction was performed by means of bovine pericardial patch. Two patients received homograft conduit, in seventeen cases a biological prosthesis was implanted and the remaining 13 patients had a mechanical prosthesis valve graft conduit.

Mean CPB time was $226 \pm 100$ minutes (range, 109523 minutes) and the mean duration of aortic crossclamping was $170 \pm 48$ minutes (range, $96-285 \mathrm{~min}$ ).

\section{Follow-up}

Follow-up data was obtained from direct contact with patients and/or their next of kin; further information was also available from outpatient clinic letters and from referral centres.

\section{Statistical analysis}

Continuous variables were expressed as mean \pm standard deviation. Twenty-four preoperative and operative risk factors were entered into univariate analysis (standard Student's $t$-test, Mann-Whitney test, chi square or Fisher's exact test when appropriate) to determine whether any single variable influenced hospital mortality defined as pre-discharge mortality. Variables examined at univariate analysis included gender, age, chest pain, syncope, history of coronary artery disease (CAD), peripheral vasculopathy, diabetes mellitus, hypertension, preoperative chronic renal failure, chronic obstructive pulmonary disease (COPD), history of cerebral stroke, active endocarditis, redo operation, interval time admission to surgery, previous aortic root replacement (ARR) surgery, acute heart failure, cardiogenic shock, preoperative inotropes, preoperative mechanical ventilation, diagnosis of $\mathrm{CAD}$ needing $\mathrm{CABG}$, unplanned $\mathrm{CABG}$, associated mitral valve (MV) surgery, $\mathrm{CPB}$ time and cross-clamping time.

Each variable reaching significance $(\mathrm{P}<0.05)$ at univariate analysis was entered into a multi-variate logistic regression model for further analysis.

Survival rates and freedom from new endocarditis/ reoperation were calculated using the Kaplan-Meyer method. Statistical analyses were performed using the Stat-View Statistical Software Package 5.0 (SAS Institute, Inc. Cary, NC, USA), NCSS 2001 (Number Chruncher Statistical System, Kaysville, Utah, USA).

\section{Results}

\section{Clinical presentation and microbiology}

All patients experienced typical signs of sepsis and in most cases, new onset/worsening dyspnoea (27/32, $84 \%)$. Particularly, 10 patients had experienced a recent episode of heart failure; two presented in cardiogenic shock and required preoperative support with inotropes and mechanical ventilation. One patient sustained a new neurologic deficit with a right hemispheric stroke resulting in a partially recovered left hemiparesis. Six patients came to the attention of the cardiologists because of chest pain, while three patients had experienced syncope. In these last cases, imaging studies revealed extensive tissue destruction, with a complete valve graft conduit detachment $(n=2)$ and the presence of valve graft conduit systolic collapse due to high pressure inside the pseudoaneurysm sac $(n=1)$.

Blood and tissue samples were sent for microbiology study: Staphylococci were found in seven patients (22\%), Streptococci in two patients and in six cases, gram negative bacteria were found (19\%). Despite repeated blood cultures, intraoperative swabs and examination of multiple specimens, endocarditis was culture-negative in seventeen patients $(53 \%)$.

\section{Anatomic findings}

In half of the cases, the discontinuity of the aortoventricular junction involved the area corresponding to the left coronary cusp/left sinus of Valsalva. In these cases, the pseudoaneurysm was contained posterior to the aorta. Eleven patients had a more lateral and anterior pseudoaneurysm arising from the area corresponding to the non-coronary or right coronary sinus of Valsalva and characterised by a wider extension, with involvement of the whole aorta in 5 cases and of the pulmonary trunk in one patient. A complete detachment of the valve graft conduit from the left ventricle was described in five patients, two of them presented with the previously implanted valve graft conduit secured by only the coronary button sutures (Figure 2).

\section{Hospital mortality}

The in-hospital mortality reached $22 \%$ (7 patients). All these patients had undergone a redo procedure- in three cases the index operation was the third surgical procedure through sternotomy.

There was one intraoperative death due to refractory RV 


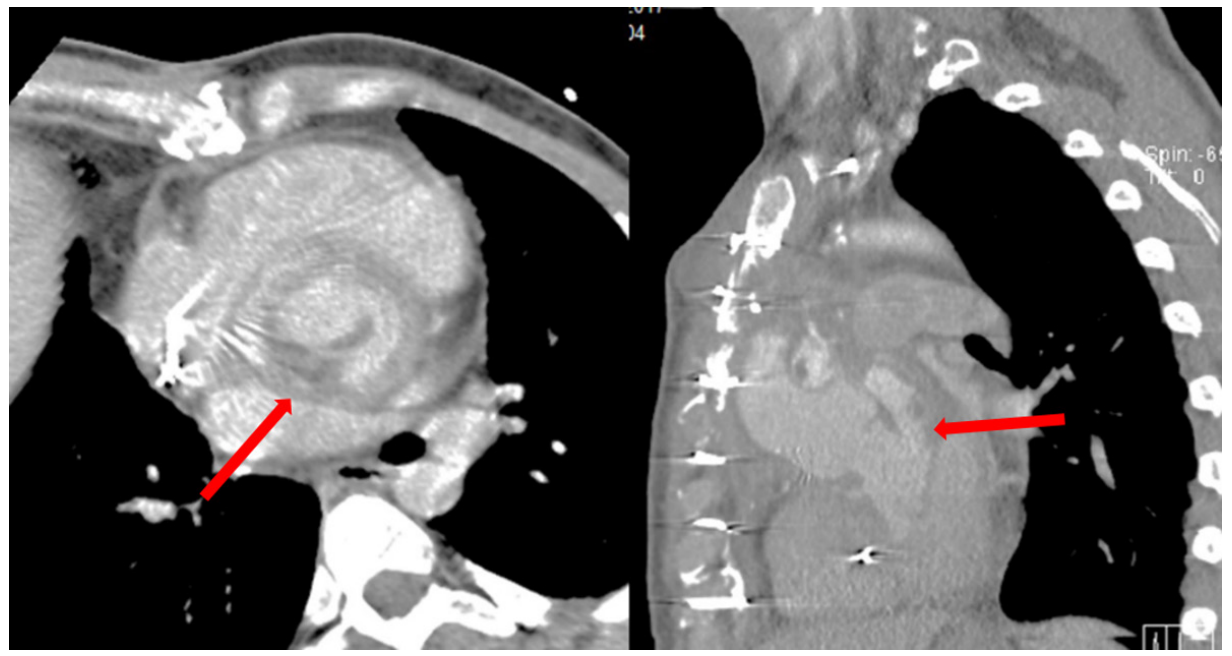

Figure 2 Multiplanar CT images showing circumferential aorto-ventricular discontinuity (red arrow).

failure after redo Bentall and extensive reconstruction of left ventricular outflow tract (LVOT) and superior caval vein. Five patients were not discharged from the intensive care unit (ICU), four of them sustained prolonged postoperative low cardiac output syndrome. Final causes of death for these patients was multi-organ failure syndrome (MOFS) in two cases, refractory $\mathrm{RV}$ failure due to perioperative myocardial infarction (MI) $(\mathrm{n}=1)$, cerebral stroke $(\mathrm{n}=1)$ and sepsis $(\mathrm{n}=1)$. A further patient did well in the initial postoperative course; he was discharged from ICU 4 days after the operation but ultimately experienced cardiac arrest on postoperative day 19 during rehabilitation.

At univariate analysis, age $(\mathrm{P}=0.029)$ and preoperative support with inotropes $(\mathrm{P}=0.035)$ were significant risk factors for hospital mortality. No significance was retained after logistic regression (age $\mathrm{P}=0.051,1.000-1.281$; preoperative inotropes $\mathrm{P}=0.058,0.910-2026)$.

The median ICU stay was 3 days (range, 1-60 days); median postoperative hospital stay was 9 days (range, 9-60 days).

\section{Hospital morbidity}

The postoperative course was complicated in 24 cases (75\%). Early reoperation for excessive bleeding or tamponade was necessary in five patients (16\%). Eighteen patients $(56 \%)$ suffered low cardiac output requiring cardiac support. New neurologic deficit was registered in six patients (19\%): cerebral stroke in one case, one patient sustained a transient ischemic accident, while four patients had delayed recovery of consciousness. Four patients (12\%) had pulmonary complications and twelve developed postoperative acute kidney injury requiring continuous renal replacement therapy in four cases. We registered one case of mediastinitis and in four cases $(12 \%)$, a permanent pacemaker implantation was necessary for complete AV block.

\section{Mid-term follow-up}

All patients were followed at the outpatient clinics of each department or at referral sites. Median follow-up time was 17 months (range, 0-92 months). We registered five deaths during this period. Four of these patients died in the first seven months from operation-in three of these cases infective complications were the main cause of death. Another patient who experienced a full recovery sustained acute myocardial infarction complicated by sudden death four years after the operation. The remaining 20 patients who were discharged from hospital did generally well and reported acceptable quality of life. Three of them required further hospital admissions. One patient had recurrent aortic valve prosthesis endocarditis 2 years after the index operation, he underwent medical therapy with antibiotics, and he was asymptomatic at 4-year follow-up from this episode. The second patient, known to have preoperative moderate to severe LV systolic dysfunction, experienced an episode of heart failure one year after the operation; imaging and clinical investigations ruled out a new cardiac problem or infective aortic complications. A third young 


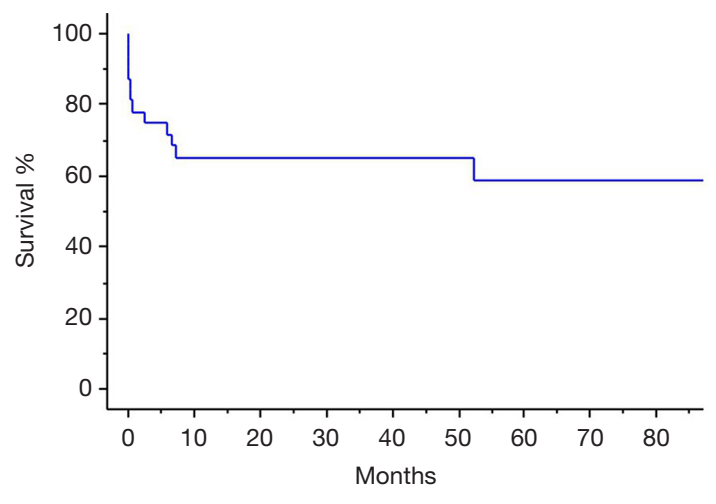

$\begin{array}{llllll}\text { Pts. at risk } 32 & 18 & 14 & 13 & 10 & 8\end{array}$

Figure 3 Kaplan-Meier survival curves from surgical operation.

patient underwent redo aortic root replacement three times for recurrence of aortic false aneurysm from the proximal anastomosis site; ultimately, he received heart transplant and, at 1-year follow-up from this last procedure, he is experiencing a satisfactory quality of life, being independent and active.

Overall survival (including hospital mortality) at 1-year and 5 -year follow-up was $65 \%$ and $59 \%$ respectively (Figure 3). Freedom from cardiac reoperation and recurrent endocarditis was $96 \%$ at 1 -year and $89 \%$ at 5 -year.

\section{Discussion}

Infective valve endocarditis has an estimated annual incidence of 3 to 9 cases per 100,000 persons in industrialized countries with a higher rate observed in patients with intracardiac devices (9). In contemporary populations of patients, in-hospital mortality is reported in up to $20 \%$, with an increased risk of in-hospital death in patients with prosthetic valve endocarditis and staphylococcal infection (10). Prosthetic valve endocarditis is not uncommon and accounts for $20 \%$ of all the cases of infective endocarditis (11). Its incidence in patients who had aortic valve replacement is estimated at $0.57 \%$ per personyear with the highest risk in the first year from surgery (12) and it is often associated (50-60\% of the cases) with periannular complications, such as formation of abscess, fistula or pseudoaneurysm. Surgical correction is commonly required in these cases. Several papers investigated patient outcomes after such complex procedures and a high inhospital mortality rate has been reported $(3,4)$. Comparison of surgical results is difficult because of the general lack of anatomic details depicting the degree of the severity of annulus and aortic root involvement. This heterogeneity is further underlined by the inclusion of different types of surgical procedures from aortic valve replacement (AVR)with or without patch reconstruction, to extensive repair and full root replacement. In this regard, the studies presenting precise data of location and extension of periannular complications invariably report the need for complex root surgery in case of larger abscess cavities, involvement of aorto-mitral continuity or circular detachment of the aorta $(3,4,13)$. An early mortality rate around $20 \%$ can be expected in these cases (3-5,7,13-15). Surgical complexity accounts partially for this finding since patients with aortoventricular discontinuity present usually in a poor clinical condition, with signs of heart failure and sepsis. In our series, only three patients were relatively asymptomatic, in most of the cases severe functional impairment and fever were associated with signs of mechanical complications such as chest pain or syncope due to vascular graft systolic collapse.

The optimal timing of surgery is a matter of debate. Some surgeons prefer an emergent or urgent operation to avoid further progression of tissue disruption and sudden complications, while other specialists favour an initial optimisation of the clinical picture with a longer preoperative antibiotic therapy and heart failure control. Our patients were all operated at the index hospital admission; in more than $60 \%$ of the cases, operation was performed within 5 days of admission. No impact on hospital mortality was found according to the interval time between admission and surgery. With all the limitations of our numbers, our experience supports previous reports about aortic root abscess surgery $(2,4,6,14,16)$ and follows the most recent guidelines $(17,18)$. Both European Society of Cardiology (ESC) and American Heart Association (AHA)/American College of Cardiology (ACC) papers, despite a different definition of the timing categories, provide IB recommendation for early surgery (within a few days) in these kind of cases of complicated native and prosthetic valve endocarditis presenting with tissue disruption, false aneurysm and heart failure symptoms.

All the patients underwent full root replacement and this is the common choice in the presence of annular discontinuity and invasion outside the aortic root- especially in prosthetic valve endocarditis. In thirteen patients, the severity of annular destruction and the need for an extensive radical debridement required annular/periannular patch reconstruction before the implantation of the valve graft conduit. Some authors advocate the use of homograft 
conduit in these cases $(19,20)$. Homograft is viable tissue and is expected to be more resistant to re-infection $(19,20)$. Furthermore, it can ease tissue adaptation and reduce residual dead space. However, prompt availability might be a concern in urgent/emergent cases, and the expected homograft structural valve deterioration and calcification poses the risk of a new, challenging reoperation $(20,21)$. Only two patients in our series received a full root replacement with an allograft; a bioprosthesis was chosen in seventeen patients while the remaining thirteen patients received a mechanical conduit (mean $\pm \mathrm{SD}, 59.71 \pm 17.9$ vs. $59.54 \pm 10.73$, respectively; $\mathrm{P}=0.97$ ). We found no difference in terms of mortality between these two groups of patients. Decision about the type of conduit was based mainly on surgeon preference as there is no clear evidence and no recommendation supporting any particular choice between different types of conduits and valves in aortic root/valve endocarditis $(17,18,22)$.

These time-consuming procedures present a challenge in terms of providing safe isolation of mediastinal structures, an effective radical debridement and tissue reconstruction. Myocardial protection is crucial for the early outcome; alongside careful cardioplegic protection, we have always put great effort into preventing ventricular distension, coronary embolization of debris and thrombi and suboptimal restoration of coronary flow. Re-attachment of coronary ostia can be difficult in these cases because of adhesions and the frailty of the aortic wall: we were able to reimplant coronary ostia in all but two patients who required unplanned CABG and were successfully weaned off $\mathrm{CPB}$. However, postoperative low cardiac output syndrome was common and as extensively reported in the literature $(4,13)$, characterized the course of patients who did not survive the operation. Long CPB and cross-clamp time in decompensated and septic patients are poorly tolerated, thus, it is not surprising that need for preoperative inotropes was a significant risk factor, with CPB time nearly reaching significance for early mortality at univariate analysis.

The early months after the operation are still burdened by a significant mortality risk; we registered four deaths in the first 7 months after the surgical procedure. In these cases, a partial recovery and pulmonary/systemic reinfection were the main cause of death.

An argument either way can be made regarding offering such an invasive and risky surgery to patients presenting in a poor preoperative clinical status. Medical therapy alone provides dismal results in patients with aortic valve endocarditis and periannular complications who refused or were denied a surgical option. While on medical therapy, major adverse events were reported in up to $70 \%$ of the cases in the first 3 years (1), with significant risk of events such as chronic heart failure, need for urgent operation and sudden death in the first weeks/months after the diagnosis (23). Our mid-term follow-up data showed an acceptable survival and freedom from reoperation; our 5 -year survival of $59 \%$ similarly aligned with other centres' experience of $50-60 \%(4,5,14,16)$. Furthermore, we were able to demonstrate that most of the patients discharged from the index operation are in general good condition, active and autonomous. For all these reasons, we believe that a surgical option should be always considered in these cases despite the expectation of intraoperative technical difficulties and the need of a prolonged postoperative recovery. Complex proximal aortic surgery represents the only proper treatment able to provide (in association with a careful postoperative medical management) satisfactory mid-term survival and quality of life.

\section{Acknowledgments}

None.

\section{Footnote}

Conflicts of Interest: The authors have no conflicts of interest to declare.

\section{References}

1. Anguera I, Miro JM, San Roman JA, et al. Periannular complications in infective endocarditis involving prosthetic aortic valves. Am J Cardiol 2006;98:1261-8.

2. Chen GJ, Lo W, Tseng H, et al. Outcome of surgical intervention for aortic root abscess: a meta-analysis. Eur J Cardiothorac Surg 2018;53:807-14.

3. Baumgartner FJ, Omari BO, Robertson JM, et al. Annular abscesses in surgical endocarditis: anatomic, clinical, and operative features. Ann Thorac Surg 2000;70:442-7.

4. Leontyev S, Davierwala PM, Krogh G, et al. Early and late outcomes of complex aortic root surgery in patients with aortic root abscesses. Eur J Cardiothorac Surg 2016;49:447-54.

5. Takahashi H, Arif R, Kallenbach K, et al. Surgical treatment of aortic valve endocarditis with left ventricularaortic discontinuity. Ann Thorac Surg 2013;96:72-6.

6. Malvindi PG, van Putte BP, Heijmen RH, et al. 
Reoperations for Aortic False Aneurysms After Cardiac

Surgery. Ann Thorac Surg 2010;90:1437-43.

7. Graupner C, Vilacosta I, San Roman JA, et al. Periannular extension of infective endocarditis. J Am Coll Cardiol 2002;39:1204-11.

8. Feuchtner GM, Stolzmann P, Dichtl W, et al. Multislice computed toography in infective endocarditis. Comparison with transesophageal echocardiography and intraoperative findings. J Am Coll Cardiol 2009;53:436-44.

9. Hoen B, Duval X. Infective endocarditis. N Engl J Med 2013; 368:1425-33.

10. Murdoch DR, Corey GR, Hoen B, et al. Clinical presentation, etiology, and outcome of infective endocarditis in the 21st century: the International Collaboration on Endocarditis-Prospective Cohort Study. Arch Intern Med 2009;169:463-73.

11. Wang A, Athan E, Pappas PA, et al. Contemporary clinical profile and outcome of prosthetic valve endocarditis. JAMA 2007;297:1354-61.

12. Glaser N, Jackson V, Holzmann MJ, et al. Prosthetic Valve Endocarditis After Surgical Aortic Valve Replacement. Circulation 2017;136:329-31.

13. Wilbring M, Tugtekin M, Alexiou K, et al. Composite aortic root replacement for complex prosthetic valve endocarditis: initial clinical results and long-term follow-up of high-risk patients. Ann Thorac Surg 2012;94:1967-74.

14. Grubitzsch H, Tarar W, Claus B, et al. Risks and challenges of surgery for aortic prosthetic valve endocarditis. Heart Lung Circ 2018:27:333-43.

15. Esaki J, Leshnower BG, Binongo JN, et al. Reoperative aortic root replacement: outcome in a contemporary series. J Thorac Cardiovasc Surg 2017;154:800-808.e3.

Cite this article as: Malvindi PG, Mikus E, Caprili L, Santarpino G, Margari V, Calvi S, Nasso G, Gregorini R, Carbone C, Albertini A, Speziale G, Paparella D. Aortic valve endocarditis complicated by proximal false aneurysm. Ann Cardiothorac Surg 2019;8(6):667-674. doi: 10.21037/ acs.2019.05.17
16. Okada K, Tanaka H, Takahashi H, et al. Aortic root replacement for destructive aortic valve endocarditis with left ventricular-aortic discontinuity. Ann Thorac Surg 2008;85:940-5.

17. Habib G, Lancellotti P, Antunes MJ, et al. 2015 ESC Guidelines for the management of infective endocarditis. Eur Heart J 2015;36:3075-128.

18. Nishimura RA, Otto CM, Bonow RO, et al. 2017 AHA/ ACC Focused Update of the 2014 AHA/ACC Guideline for the Management of Patients With Valvular Heart Disease: A Report of the American College of Cardiology/ American Heart Association Task Force on Clinical Practice Guidelines. J Am Coll Cardiol 2017;70:252-89.

19. Preventza O, Mohamed AS, Cooley DA, et al. Homograft use in reoperative aortic root and proximal aortic surgery for endocarditis: A 12-year experience in high-risk patients. J Thorac Cardiovasc Surg 2014;148:989-94.

20. Musci M, Weng Y, Hübler M, et al. Homograft aortic root replacement in native or prosthetic active infective endocarditis: twenty-year single-center experience. J Thorac Cardiovasc Surg 2010;139:665-73.

21. Malvindi PG, van Putte BP, Leone A, et al. Aortic reoperation after freestanding homograft and pulmonary autograft root replacement. Ann Thorac Surg 2011;91:1135-40.

22. Jassar AS, Bavaria JE, Szeto WY, et al. Graft selection for aortic root replacement in complex active endocarditis: does it matter? Ann Thorac Surg 2012;93:480-7.

23. Chan KL. Early clinical course and long-term outcome of patients with infective endocarditis complicated by perivalvular abscess. CMAJ 2002;167:19-24. 